\title{
Danjon Number and RGB-colors in Total Lunar Eclipse: 2000-2017
}

\section{Número de Danjon y color RGB en los Eclipses Totales de Luna: 2000-2017}

\author{
Falcon, Nelson ${ }^{1}$; Ortega, Alcides ${ }^{1}$ \\ 1. Laboratorio de Física de la Atmósfera y Espacio Ultraterrestre, Universidad de Carabobo, Facultad \\ Experimental de Ciencias y Tecnología FACYT , Dpto. de Física, Valencia Edo. Carabobo Venezuela. \\ (*) E-mail: nelsonfalconv@gmail.com; aortega@uc.edu.ve
}

Received: 03/04/2018 Accepted: 03/07/2018

DOI: $10.7149 /$ OPA.51.3.50025

\begin{abstract}
:
The apparent magnitude and chromaticity of the lunar disk during the maximum phase of a total eclipse of the Moon inform about the atmospheric optical thickness and the average column density of aerosols in the Earth's atmosphere. The estimation of the magnitude of the total lunar eclipse has been used for paleoclimatology studies and has been correlated with global volcanic activity; hence the importance of its quantification. However, the qualitative scale used to determine the magnitude and chromaticity, known as Danjon's number, is not sufficient to unambiguously characterize these magnitudes. A methodology is sought to determine a well-defined scalar, which links the Danjon number to the RGB digital color of the photographs, called the Danjon Factor (FD); and the results obtained for all the total eclipses of Luna occurred in the period 2000-2017. It is concluded that the Danjon Factor is an effective measure of the magnitude and chromaticity calculable through the digital RGB color in the Total Lunar Eclipses.
\end{abstract}

Key words: Danjon number, Total Lunar eclipse, digital colors, atmospheric optical thickness

\section{RESUMEN:}

La magnitud aparente y la cromaticidad del disco lunar durante la fase máxima de un eclipse total de Luna informan sobre el espesor óptico atmosférico y la densidad media columnar de aerosoles en la atmósfera terrestre. La estimación de la magnitud del eclipse lunar total se ha empleado para estudios de paleoclimatología y se ha correlacionado con la actividad volcánica global; de allí la importancia de su cuantificación. Sin embargo la escala cualitativa usada para determinar la magnitud y cromaticidad, conocida como número de Danjon, no es suficiente para caracterizar unívocamente estas magnitudes. Se propone una metodología para determinar un escalar bien definido, que vincula el número de Danjon al color digital RGB de las fotografías, denominado Factor de Danjon (FD); y se presentan los resultados obtenidos para todos los eclipses totales de Luna acaecidos en el periodo 2000-2017. Se concluye que el Factor de Danjon es una medida eficaz de la magnitud y cromaticidad calculable a través del color digital RGB en los Eclipses Totales de Luna.

Palabras clave: Número de Danjon, Eclipse Total de Luna, Color digital, espesor óptico atmosférico

\section{REFERENCES AND LINKS / REFERENCIAS Y ENLACES}

[1] J. Kepler. Astronomiae Pars Optica. Marnius, Frankfurt. pp. 267 (1604)

[2] G. Baillet, "Réfraction Atmosphérique," Observations \& Travaux 80, 22-27 (2012)

[3] L. Rayleigh Phyl Mag. XLI 107, 274-278 (1871)

[4] R.A. Keen, "Volcanic Aerosols and Lunar Eclipses," Science 222, 1011-1013 (1983)

[5] R.A. Keen, "Volcanic Aerosols optical thicknesses since 1960," Bull. Global Volcanism Network 22, 11 (1997)

[6] R.A. Keen, "Volcanic Aerosols derived from Lunar eclipse observation". Bull. Global Volcanism Network 26, 5 (2001). 
[7] R.B. Stothers, "Stratospheric Transparency Derived from Total Lunar Eclipse Colors, 1801-1881," PASP 117, 1445-1450 (2005)

[8] R.B. Stothers, "Stratospheric Transparency Derived from Total Lunar Eclipse Colors, 1665-1800," PASP ,116, 886-893 (2004)

[9] R.B. Stothers, "Three centuries of observation of stratospheric transparency,. Climatic Change 83, 515521. (2007)

[10] A. Danjon "Relation Entre l'Eclairement de la Lune Eclipsee et l'Activite Solaire," L'Astronomie. 35, 261-265 (1921)

[11] A Danjon. Astronomie generale. J. \& R. Sennac (1952)

[12] http://eclipse.gsfc.nasa.gov/OH/Danjon.html

[13] N. Hernitschek et al. "Lunar eclipse photometry: absolute luminance measurements and modeling," Applied Optics 34, 62-70 (2008)

[14] M. Vollmer et al. "Simulating irradiance during lunar eclipses: the spherically symmetric case," Applied Optics 34, 52-61 (2008)

[15] E. Hecht ; A. Zajac. Optica. Addison Wesley Delaware (1974)

[16] Computational Chemistry Comparison and Benchmark DataBase. NIST Standard Reference Database Number 101 Release 19, April 2018, Editor: Russell D. Johnson III https://cccbdb.nist.gov/diatomicexpbondx.asp

[17] W. J. McNeil; E. Murad, and S. T. Lai. "Comprehensive model for the atmospheric sodium layer," J. Geophys. Res. 8, 6847-16855. (1995)

[18] R. Muñoz;; N. Falcón,; A. Muñoz, R. Morales “Caracterización Física de la percepción de Colores Digitales," Ingeniería UC 2, 7-30 (1999)

\section{Introducción}

Cuando la Luna se introduce completamente en el cono de sombra de la Tierra; pasando a través de la umbra; tiene lugar la ocultación o eclipse total de Luna. Cabría esperar que la Luna quedara totalmente opacada e invisible al transitar por el cono de la umbra (figura 1) puesto que la longitud del cono de sombra de la Tierra; del orden de 217 veces el Radio Terrestre; es mucho mayor que la distancia Terra-Luna, del orden de 60 veces el radio terrestre.

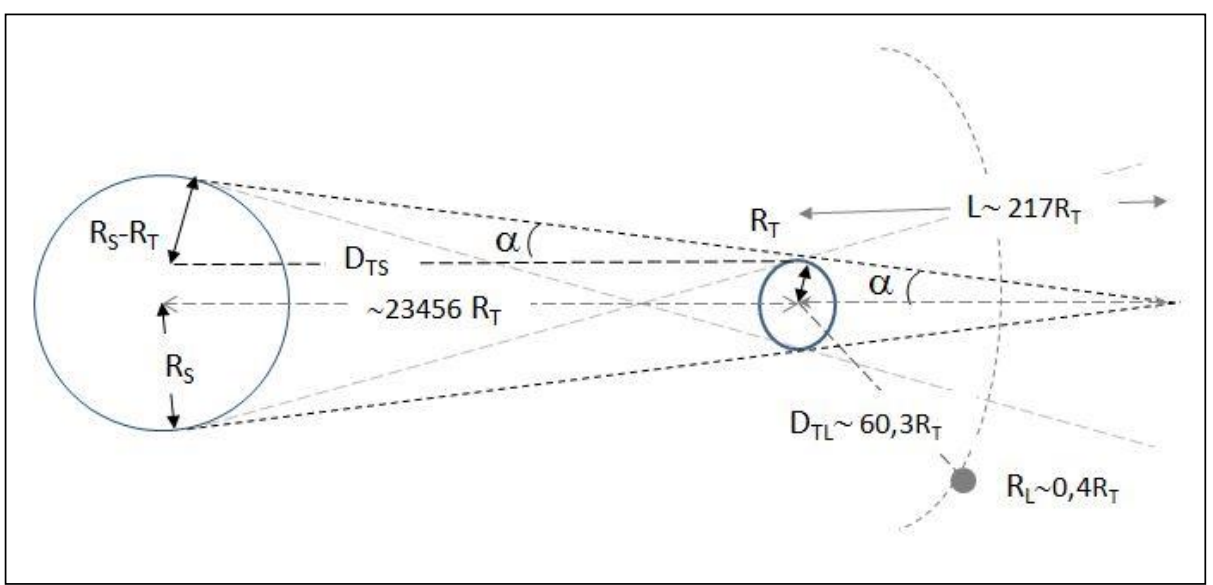

Fig. 1. Geometría de un Eclipse Lunar sin considerar la atmósfera terrestre. Donde $R_{S} R_{T}$ y $R_{L}$ representan los radios medios del Sol, la Tierra y la Luna respectivamente, DTS y $D_{\text {TR }}$ las distancias medias Tierra-Sol y Tierra-Luna, L es la longitud del cono de sombra y $\alpha \sim 4,610^{-3}$ rad., el ángulo generatriz del cono de sombra.

Sin embargo la Luna muestra una magnitud visual aparente $(m=-4 a+4)$ y una coloración opaca en la región visible del espectro electromagnético (400-750 nm), que varía en cada eclipse particular, desde un ocreamarillo hasta un rojo oscuro. Esta coloración y brillo de la Luna eclipsada es debida a desviación de los rayos solares en la atmósfera terrestre, hasta penetrar en el cono de sombra de la Tierra y proyectarse sobre el disco lunar (figura 2) como lo señaló primeramente Kepler [1]. 


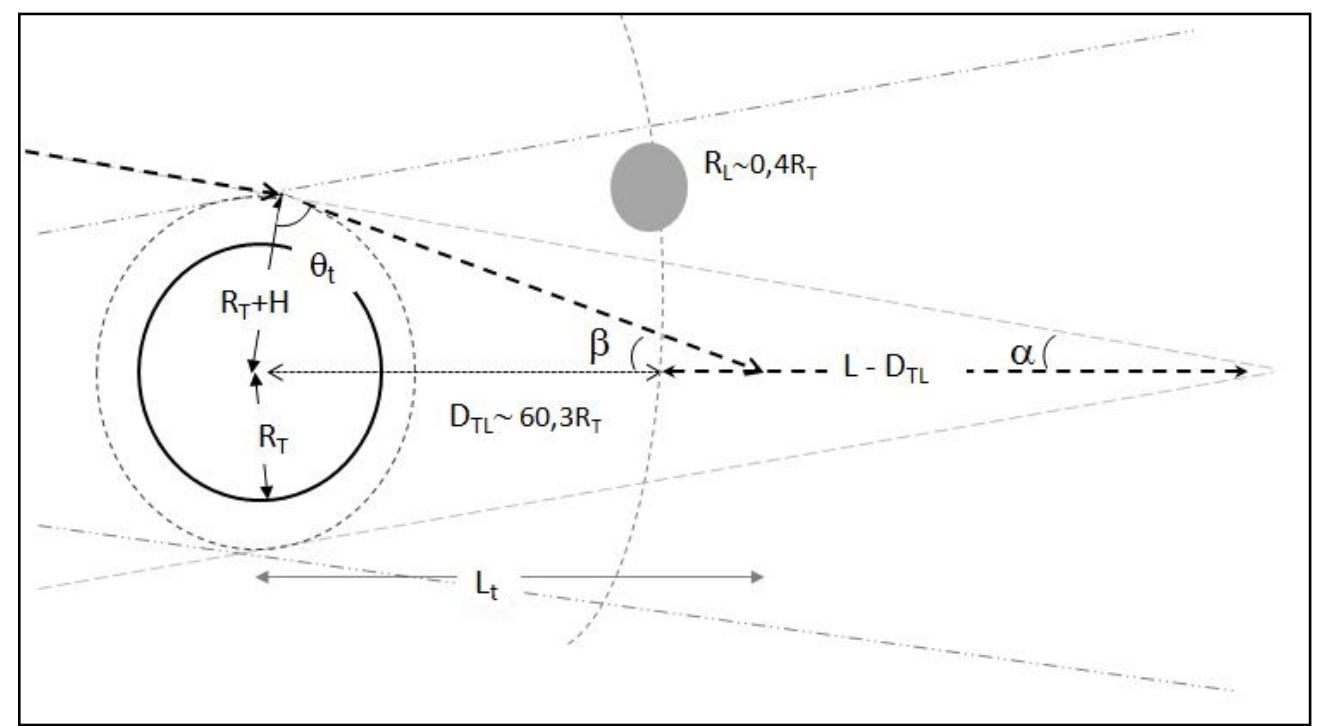

Fig. 2. Desviación de los rayos solares en un Eclipse Lunar por la atmósfera terrestre. Donde H representa la altura media de la atmósfera, $\mathrm{L}_{\mathrm{t}}$ la longitud del cono de luz transmitida, $\theta_{\mathrm{t}}$ el ángulo de refracción por la atmósfera y $\beta$ el ángulo generatriz del cono de de luz transmitido.

La luz solar, en la región visible del espectro electromagnético, es atenuada, dispersada y refractada al pasar por la atmósfera terrestre. La concentración y tamaño de los constituyentes atmosféricos difractan los rayos solares en mayor o menor grado, ocasionando el cromatismo y luminosidad de la Luna eclipsada durante la fase de totalidad. Un cálculo elemental de la circunstancia de un Eclipse total de Luna a través de la figura 2 , muestra que la longitud del cono de la luz solar refractada $\left(\mathrm{L}_{\mathrm{t}}\right)$ por la atmósfera terrestre es:

$$
\frac{L_{t}}{\operatorname{sen}\left(\theta_{t}\right)}=\frac{R_{T}+H}{\operatorname{sen}(\beta)} \Rightarrow L_{t}=\frac{R_{T}+H}{\eta} \frac{1}{\operatorname{sen}(\beta)}
$$

Donde se ha usado la ley de Snell para incorporar el índice de refracción relativo $\eta$, entre la atmósfera y el vacío. De la geometría en la figura 2 , se verifica que $\operatorname{sen}(\beta) \simeq \cos \left(\theta_{t}\right)+\alpha \operatorname{sen}\left(\theta_{t}\right)$, puesto que $\alpha<<1$; y en consecuencia la relación (1) puede ser expresada como:

$$
L_{t}=\frac{R_{T}+H}{\alpha+\sqrt{\eta^{2}-1}}
$$

Para la atmósfera terrestre estándar el índice de refracción medio es $\eta \cong 1,0003$ [2], y obtenemos que el cono de sombra tiene apenas una longitud del orden de 40,8 $R_{T}$ (menor que la distancia Tierra-Luna: $60 R_{t}$ ) y por lo tanto no se obtendrían eclipses lunares, en contradicción de lo observado. Es claro entonces que no se puede usar el índice de refracción medio de la atmósfera en la relación (2). En general el índice de refracción depende de la longitud de onda y su cálculo preciso debe hacerse a través de la relación de dispersión, como se discutirá en la próxima sección. La dispersión de la luz por los gases, particularmente de la luz solar en la atmósfera terrestre fue estudiada primeramente por Lord Rayleigh [3], mostrando que la sección total de dispersión $\left(\sigma \cong \pi \mathrm{r}_{0}{ }^{2}\right)$ es proporcional a la cuarta potencia de la longitud de onda; y en consecuencia explica el color azul del cielo debido a que las longitudes de onda corta (azul) son mas dispersadas que las longitudes de onda larga (rojo) en la región visible del espectro electromagnético. La atenuación y dispersión de la luz solar tiene lugar principalmente en las primeras cuatro capas atmosféricas (tropósfera, estratósfera, mesósfera y termósfera), dependiendo del radio medio de las los centros dispersivos ( $\left.\mathrm{r}_{0}\right)$ en relación a la longitud de onda incidente $(\lambda)$. Si las partículas son tales que sus dimensiones resultan mucho menores que la longitud de onda, la dispersión es de tipo Rayleigh difuminando la luz azul y azul-verdosa en el cielo, si los tamaños de las partículas son comparables a $\lambda$ (vapor de agua, humo y polvo) tiene lugar la dispersión de Mie atenuando la intensidad de la luz trasmitida; y en el caso de que las partículas tenga un tamaño mayor (polvo troposférico: volcánico y antropogénico) la absorción y oscurecimiento es independiente de la longitud de onda, oscureciendo tanto el disco lunar eclipsado como el fondo mismo del cielo. 
Como el brillo de la Luna durante el máximo del eclipse es función de la cantidad de aerosoles en la estratósfera, se han empleado las medidas de intensidad de brillo como indicador de la profundidad óptica estratosférica y para estimar el promedio de aerosoles de origen volcánico en escalas de tiempo geológico [4-8] e incluso se han usado los datos de los eclipses lunares como marcador del cambio climático global con fines paleo-meteorológicos [9].

En todos estos trabajos se estima el brillo lunar a través del número de Danjon (L). Esta escala o número de Danjon, es una medida cualitativa desarrollada a principios del siglo pasado por el astrónomo francés André-Louis Danjon [10-11] que varía desde L=0 para eclipse muy oscuro con el disco lunar apenas visible; L=1 para eclipse oscuro gris a marrón; L=2 para un disco lunar rojo intenso, L=3 para un eclipse rojo brillante con anillo amarillo en la umbra y L=4 para un eclipse muy brillante color rojo-cobre o naranja [12].

Para minimizar los errores en la valuación de la magnitud del eclipse es necesario redefinir la escala de Danjon empleando un nuevo método cuantitativo; sin embargo hasta ahora no se han propuesto escalas que permitan su valoración a partir de la fotografía digital durante el máximo del eclipse [13-14]. Las nuevas tecnologías del procesamiento digital de imágenes pueden servir para evaluar el cromatismo y magnitud del eclipse basado en los valores RGB (componentes rojo, verde y azul) de las imágenes captadas por diferentes observadores.

El objetivo del presente trabajo es desarrollar una metodología y criterios cuantitativos que permitan redefinir el Número de Danjon a partir del procesamiento digital de imágenes, con el fin ulterior de usar la magnitud de los eclipses lunares para evaluar el espesor óptico atmosférico y sus variaciones en diferentes épocas, vinculables a los cambios de la atmósfera local en escala de tiempo comparables al cambio climático global. Para ello se discuten las circunstancias geométricas de los eclipses lunares en la aproximación de rayos paraxiales incidentes sobre una atmósfera plana y la relación de dispersión (sección 2), se presenta la metodología del procesamiento digital de las imágenes en la sección 3, los resultados de la valuación cuantitativa del color y magnitud de los 17 Eclipses Totales de Luna en el periodo 2000-2017, junto a la propuesta de una escala numérica de Danjon a partir de la cromaticidad digital (plano RG) se muestra en la sección 4 y finalmente las conclusiones (sección 5).

\section{Modelo de atmósfera plana y relación de dispersión}

El cálculo preciso del índice de refracción del medio puede hacerse en una aproximación semi-clásica usando modelo de Drude-Lorentz, que se conoce como la relación de dispersión [15], que en función de la longitud de onda $(\lambda)$ puede expresarse como:

$$
\eta^{2}(\lambda)=1+\left(\frac{\lambda_{0}}{\lambda_{p}}\right)^{2}\left[1-\left(\frac{\lambda_{0}}{\lambda}\right)^{2}\right]^{-1}
$$

Donde

$$
\lambda_{0} \equiv \frac{2 \pi c}{e} \sqrt{4 \pi \varepsilon_{0} m_{e} r_{0}^{3}} \quad \lambda_{p} \equiv \frac{2 \pi c}{e} \sqrt{\frac{\varepsilon_{0} m_{e}}{N}}
$$

Siendo $m_{e}$ y $e$ la masa y carga del electrón respectivamente, $c$ la velocidad de la luz en el vacío $\varepsilon_{0}$ la permitividad eléctrica del vacío, $N$ el número de partículas por unidad de volumen y ro el radio medio de dispersión (scattering) o dimensiones de las partículas. La relación de dispersión es válida para describir las propiedades ópticas de la troposfera y estratósfera donde los momentos magnéticos de la mayoría de las moléculas son despreciables comparados con los momentos dipolares eléctricos.

La relación de dispersión (3) muestra que la componente de la luz blanca transmitida por las partículas de radio dispersor $r_{0}$, son tales que $\lambda>\lambda_{0}$, para los cuales el índice de refracción efectivo $\eta$ resulta una magnitud real. Reemplazando (3) en (2) tenemos que la longitud del cono de umbra que eclipsa a la Luna depende de la longitud de onda $(\lambda)$, del tamaño $\left(\mathrm{R}_{0}\right)$ y de la densidad del número de partículas $(\mathrm{N})$ presentes en la atmósfera: 


$$
L_{t}=\frac{R_{T}+H}{\alpha+\left(\frac{\lambda_{0}}{\lambda_{p}}\right)\left[1-\left(\frac{\lambda_{0}}{\lambda}\right)^{2}\right]^{-1 / 2}}
$$

donde se han usado las definiciones (4) y el número de Avogadro $\mathrm{N}_{\mathrm{A}}$, para evaluar las longitudes de onda expresadas en nanómetros $(\mathrm{nm})$

$$
\lambda_{p} \cong 4296 \sqrt{\frac{N_{A}}{\left[N . m^{3}\right]}}\langle n m\rangle \quad \lambda_{0} \cong 15231 \sqrt{N_{A}\left[\frac{r_{0}}{m}\right]^{3}}\langle n m\rangle
$$

La dispersión o scattering de los fotones por las partículas y moléculas atmosféricas está gobernada por la mínima distancia de acercamiento al centro dispersivo r0 que es del orden del radio de las partículas o centros dispersores. En el caso de moléculas diatómicas gaseosas resulta del orden $2 \AA$ A para el caso del sodio diatómico la longitud de enlace es del orden de 3,1 Å y del orden de 2,3 Å para $\mathrm{NaCl}$ [16]. Estos últimos son importantes debido a la capa de sodio de la estratósfera [17] situada a unos $90 \mathrm{~km}$ de altitud. El parámetro ro limita la frecuencia de natural de oscilación de las partículas que ejercen un momento dipolar eléctrico sobre la radiación que incide sobre ella; así $\mathrm{r}_{0}=2,0 ; 2,35 ; 2,519 ; 2,76 ; 3,0 ; 3,14$ corresponde a longitudes de onda mínima de $\lambda_{0}=358,450,500,575,589,652,700 \mathrm{~nm}$ respectivamente. Está claro en virtud de la relación de dispersión (3) que los fotones con longitud de onda menor que $\lambda_{0}$ serán dispersados en la atmósfera y no se propagarán para formar el cono de luz que ilumina el disco lunar durante el máximo del eclipse.
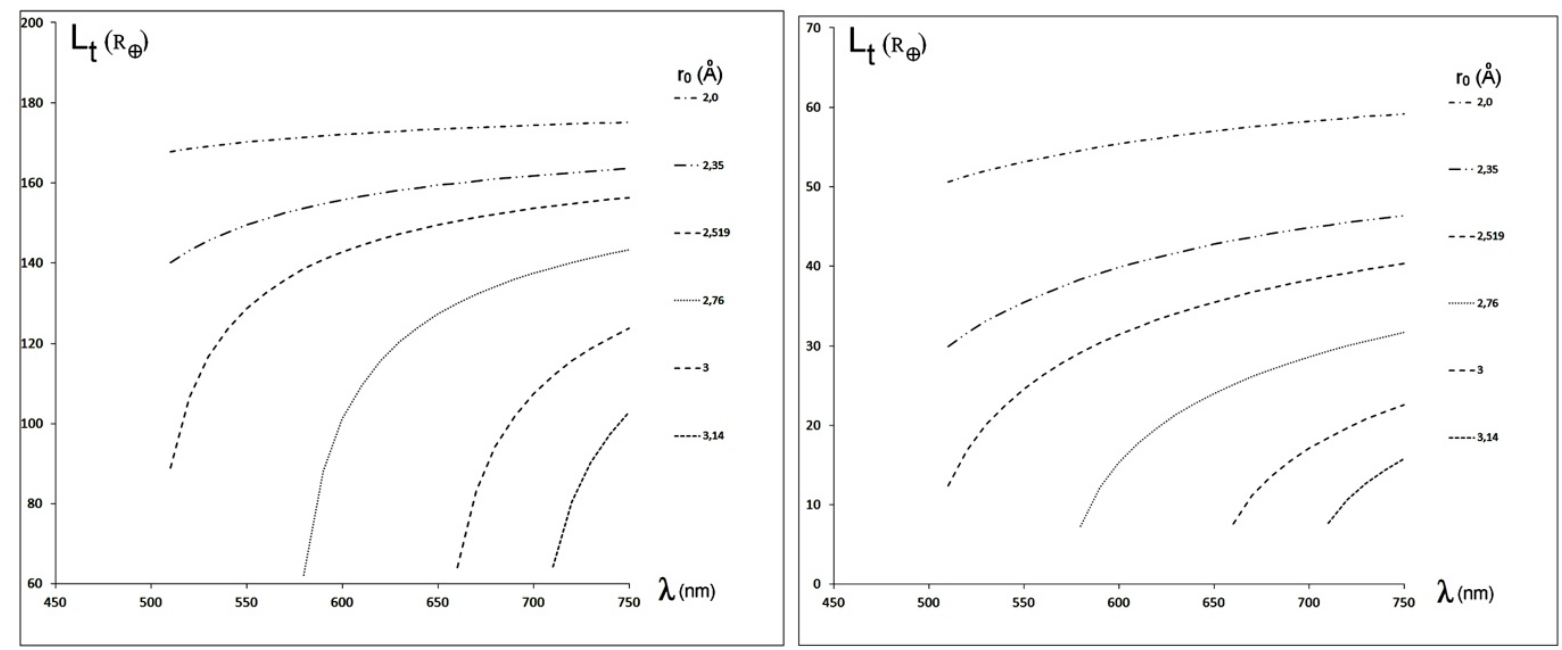

Fig. 3. Longitud del cono de luz transmitido por la atmósfera en función del parámetro ro (Izq) Número de Danjon L=4 (der) Número de Danjon L=0.

La figura 3 (Izq) muestra la longitud del cono de luz transmitido para cada una de estos centros dispersores en función de la longitudes de onda de la radiación solar visible; supuesto una concentración de partículas del orden de $\mathrm{N}=0,000125 \mathrm{~N}_{\mathrm{A}}$ (partículas/m³); obsérvese que todas las longitudes del cono de luz son mayores a $60 \mathrm{R}_{\mathrm{T}}$, y por lo tanto cabría esperar que las componentes amarillas, naranja y roja del espectro visible alcancen el disco lunar durante la fase de la totalidad; que corresponde a un eclipse muy brillante (Número de Danjon: $\mathrm{L}=4$ ). Si por el contrario se aumenta significativamente el número medio de partículas dispersoras por unidad de volumen hasta digamos la milésima parte del número de Avogadro $\left(\mathrm{N}=0,001 \mathrm{~N}_{\mathrm{A}}\right.$ partículas $/ \mathrm{m}^{3}$ ) entonces las longitudes del cono de luz transmitido, resultan menores que la distancia lunar y los fotones no alcanzarían la luna, presentándose un eclipse completamente opaco ( $\mathrm{L}=0$ en la escala de Danjon) como se observa en la figura 3 (der). Adviértase también que las componentes azul y violeta de la luz visible (de longitud de onda de orden menor a $450 \mathrm{~nm}$ ) no aparecen en el cono de luz transmitida del eclipse lunar; como es de esperar debido a la dispersión de Rayleigh en la atmósfera. 
Valores intermedios de la densidad de partículas $\mathrm{N}$ entre los casos extremos referidos, conllevarán a que algunas componentes del espectro visible sean transmitidas formando un cono de longitud mayor que la distancia lunar y otras componentes o "colores" del espectro visible, sean tales que las longitud del cono de luz transmitido y proyectado hacia la umbra tenga menor longitud que la requerida; dependiendo del parámetros $r_{0}$. Luego una escogencia adecuada de $r_{0}$ y $\mathrm{N}$ daría cuenta de magnitudes de eclipse que se corresponderían con los valores intermedios del número de Danjon ( $L=1,2$ y 3). La figura 4 representa un caso, supuesta una concentración fuera del orden de $\mathrm{N}=0,0166 \mathrm{~N}_{\mathrm{A}}$ (partículas $/ \mathrm{m}^{3}$ ); donde solo las componentes naranjas y rojas $(\lambda \geq 615 \mathrm{~nm})$ del espectro visible alcanzan el disco lunar para partículas dispersoras tales que $r_{0} \geq 2,76 \AA$; equivalente a un número de Danjon $L=2$; sin la presencia de la componente amarilla. Y análogamente, un número de Danjon $\mathrm{L}=3$ si suponemos partículas dispersoras con $\mathrm{r}_{0}<2,519 \AA$, que transmiten la componente amarilla hasta longitudes tan grandes como $120 \mathrm{R}_{\mathrm{T}}$. Si la dispersión se debiera fundamentalmente a la capa de sodio estratosférico, para el $\mathrm{N}$ considerado, se tendría $\mathrm{r}_{0} \geq 3 \AA$ transmitiendo solo las componentes rojas y el número de Danjon seria L=1. Es claro que otra escogencia adecuada de valores de $\mathrm{N}$ y ro producirá resultados similares con números de Danjon entre 1 y 3.

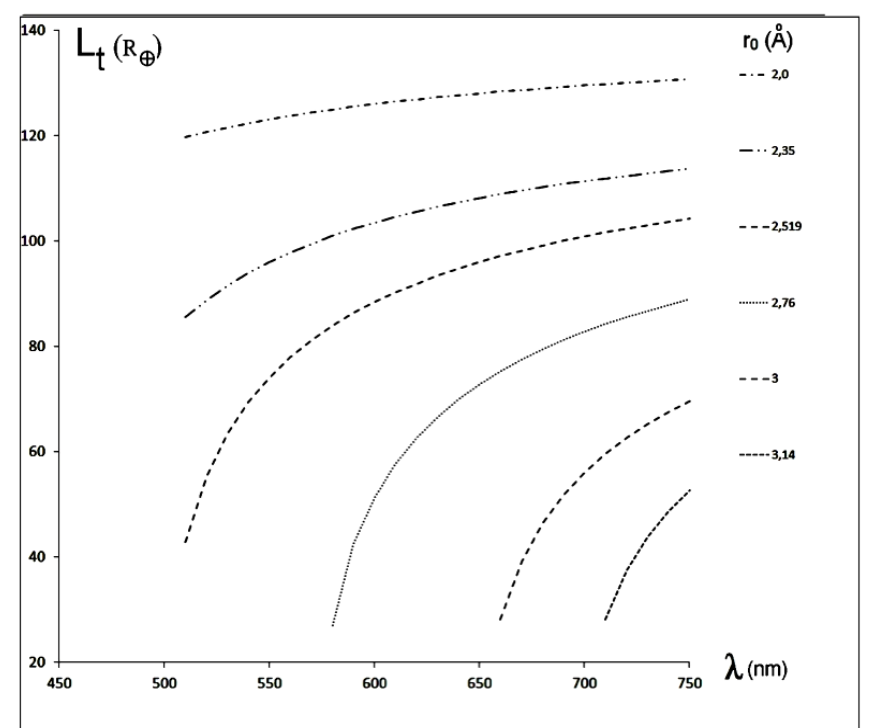

Fig. 4. Longitud del cono de luz transmitido por la atmósfera en función del parámetro ro equivalente a Número de Danjon (L): 1, 2 y 3.

\section{Procesamiento digital de color RGB}

Los valores RGB son la representación la representación del espacio de color a través de la combinación aditiva de tres colores fundamentales: rojo, verde y azul; que corresponden a las longitudes de onda 700; 546,1 y 435,8 nanómetros respectivamente. Cada componente de color se codifica por un byte que corresponde a 256 intensidades de estos colores fundamentales, en un espacio de configuración cromático con 16777216 localizaciones (figura 5). El valor de cada byte cromático en una fotografía digital dependerá de las características particulares del dispositivo empleado y se requiere que el dispositivo tenga un balance de canales RGB (rojo, verde y azul) que eviten la saturación, a fin de que el espacio cromático de la imagen sea lo más fiel posible. Las cámaras fotográficas digitales tienen un auto balance cromático; sin embargo para garantizar que los valores RGB de una imagen capturada con alguna cámara digital particular estén corregidos por el nivel de grises y blanco óptimo, se evalúan los valores RGB en las imágenes de la parcialidad del Eclipse; de modo tal que los valores RGB absolutos puedan corregirse para que ningún canal cromático alcance la saturación.

En las fotografías digitales de la Luna eclipsada, los valores cromáticos RGB variarán en cada imagen durante el desarrollo del eclipse y adicionalmente, para una misma imagen los valores RGB variarán sobre la porción del disco lunar considerada, entre otras por el albedo de los accidentes topográficos lunares. Por ello se calibran las fotografías digitales del disco lunar eclipsado en el máximo de la totalidad, sobre una muestra de seis regiones previamente seleccionadas. La selección de estas regiones no es unívoca ni limitativa, presentan la ventaja de ser accidentes lunares fácilmente identificables aun a baja intensidad lumínica, están distribuidos uniformemente sobre el disco lunar, se ubican fuera de los bordes de la Luna, y 
se conocen sus albedos particulares. La valuación se realizó sobre aéreas circulares de 314 pixeles centrados en los cráteres Copérnico, Tycho; Platón, Plinius y Grimaldi; y el área central del mar Crisium (Véase Tabla 1). Usando el software libre GIMP 2.8, se promedian los valores RGB en las áreas seleccionadas y se corrigen para el exceso de azul (figura 5); pues durante el máximo la luz del disco lunar no tiene componente azul, absorbida por la atmósfera debido a la dispersión de Rayleigh. Adicionalmente se realiza la valoración media de las componentes RGB para el disco lunar eclipsado durante el máximo con tamaño de 400 pixeles de radio.

En el espacio RGB el punto origen es el negro, el blanco de coordenadas $(255,255,255)$ es tal que su módulo de distancia es máximo 360,6. Adviértase que en el plano RG los puntos cercanos al origen son más oscuros y los más alejados son más brillantes (fig.5).

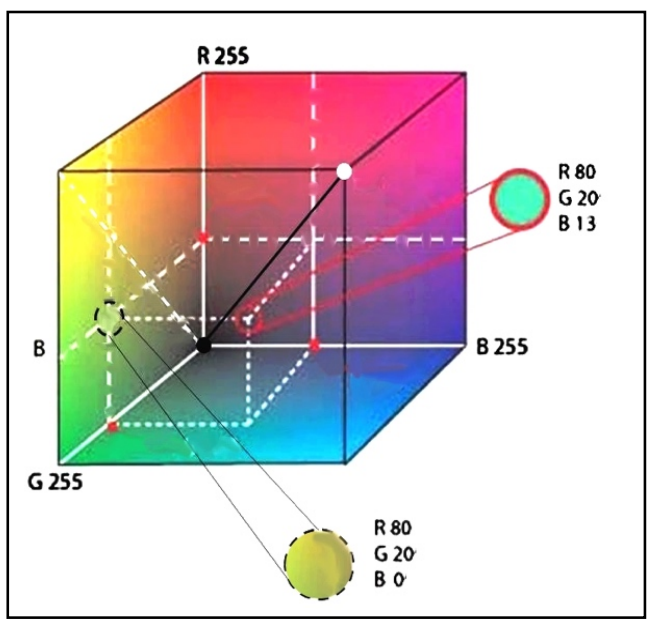

Fig. 5. Espacio cromático digital RGB y su proyección para caracterizar la luz lunar en la fase máxima del eclipse (plano RG).

En la Tabla I se muestran las componentes cromáticas promedio RGB de los accidentes lunares considerados, y del disco lunar, para los 17 Eclipses Totales de Luna ocurridos en el periodo 2000-2017 (tercera columna). La primera columna informa además sobre las regiones de visibilidad del eclipse y la referencia de las imágenes fotográficas disponibles para cada evento; en la siguiente columna la imagen digital obtenida durante el máximo de la totalidad, la autoría de la fotografía, el equipo fotográfico utilizado y foco, tiempo de exposición y nivel ISO. Y en la última columna la apariencia de un disco uniforme equivalente a los valores RGB promediados. Adviértase que la apariencia cromática del disco uniforme, aunque similar a la imagen digital, no se corresponde necesariamente a la percepción visual ordinaria puesto que la respuesta cromática del ojo humano atenúa el rojo respecto al amarillo y el verde, debido a la curva de sensibilidad espectral. De resulta que un observador a ojo desnudo percibirá los disco con cromaticidades mas amarillentas que marrones y mas naranjas que rojizas [18].

TABLA 1. Valores RGB durante el máximo de los Eclipses Totales de Luna: 2000-2017.

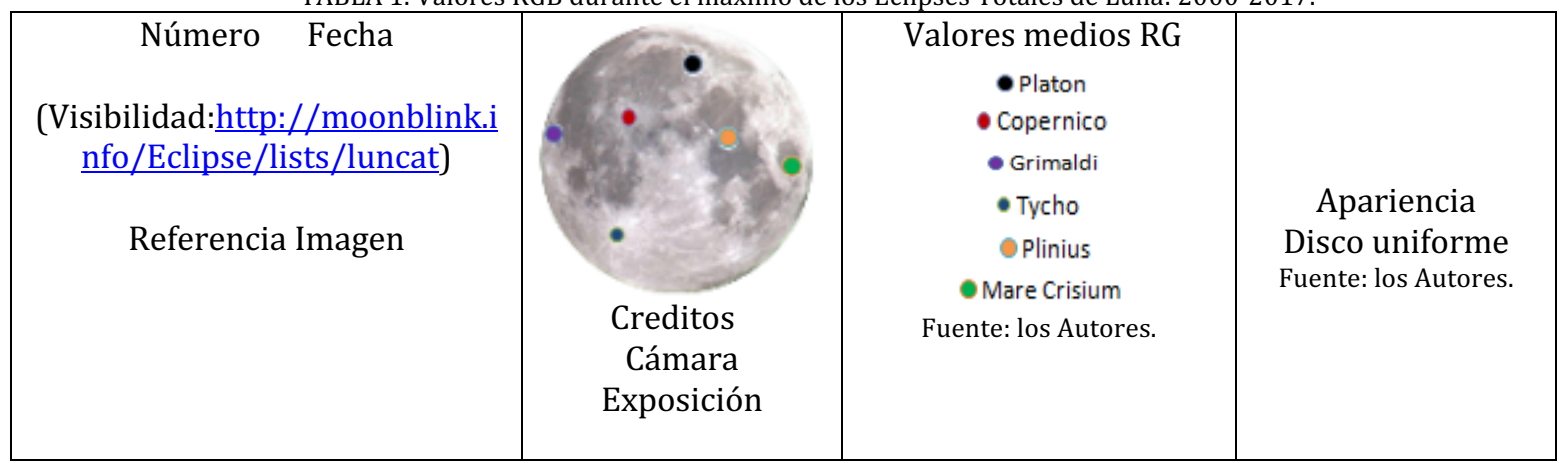




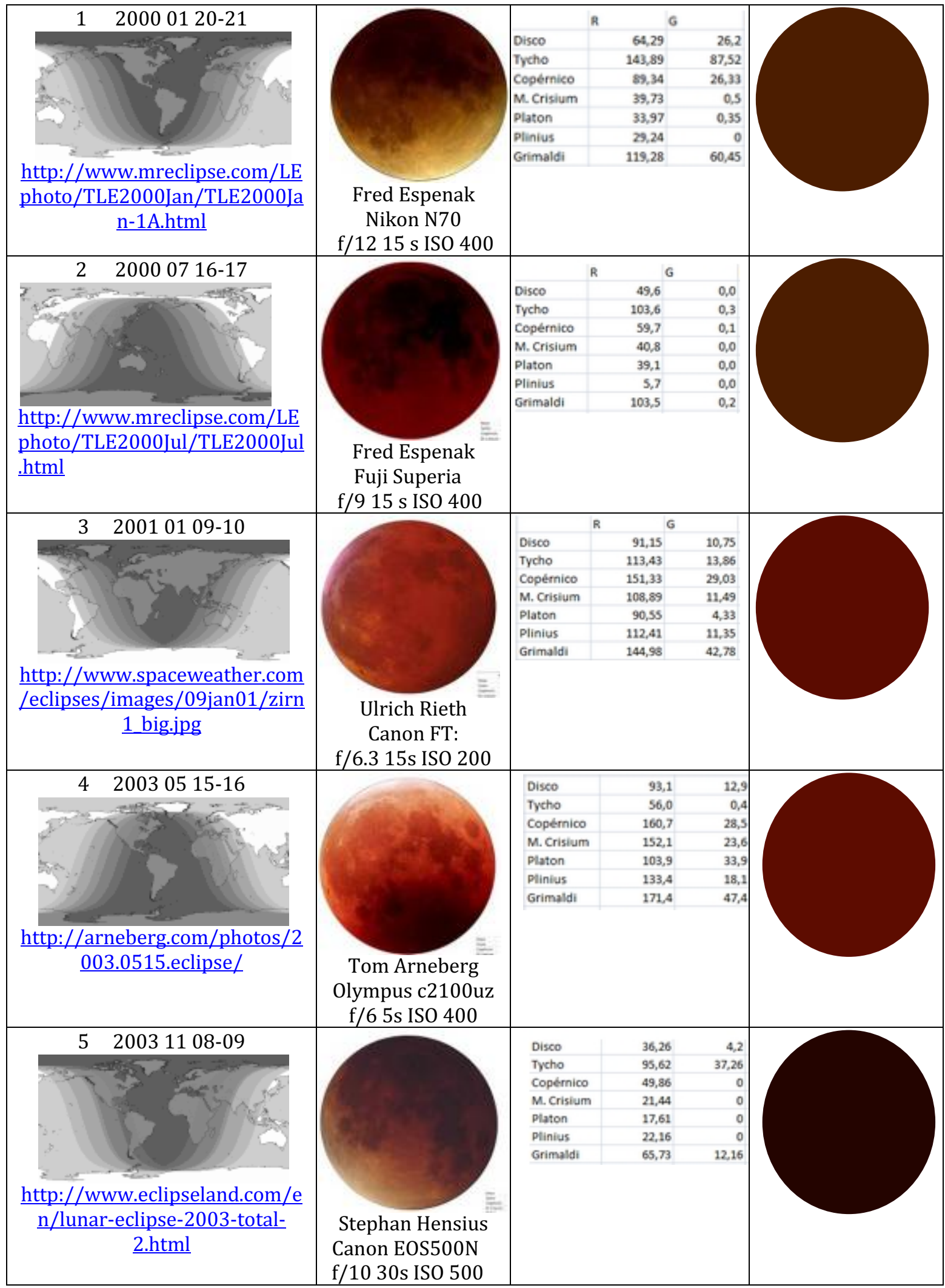




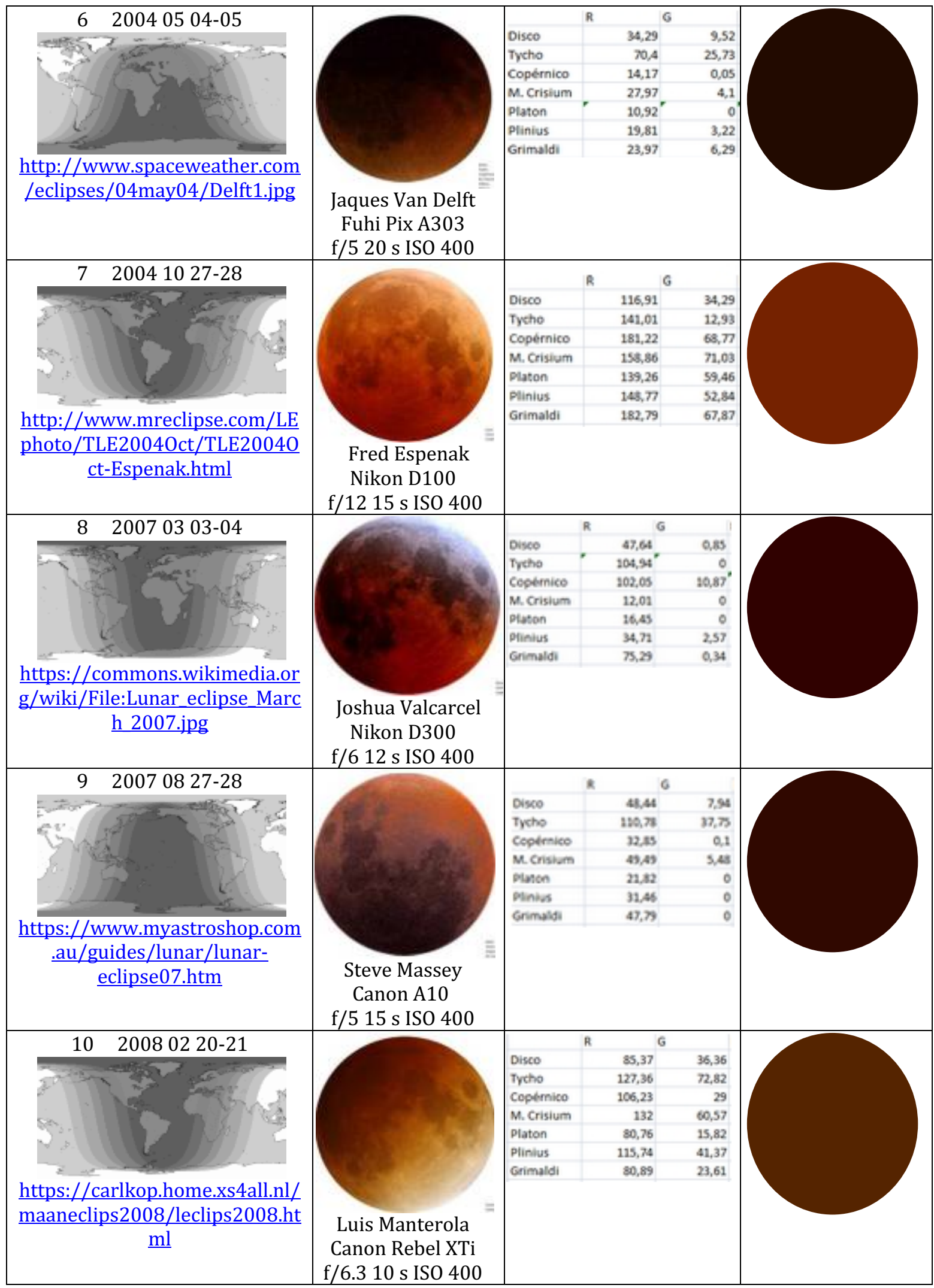




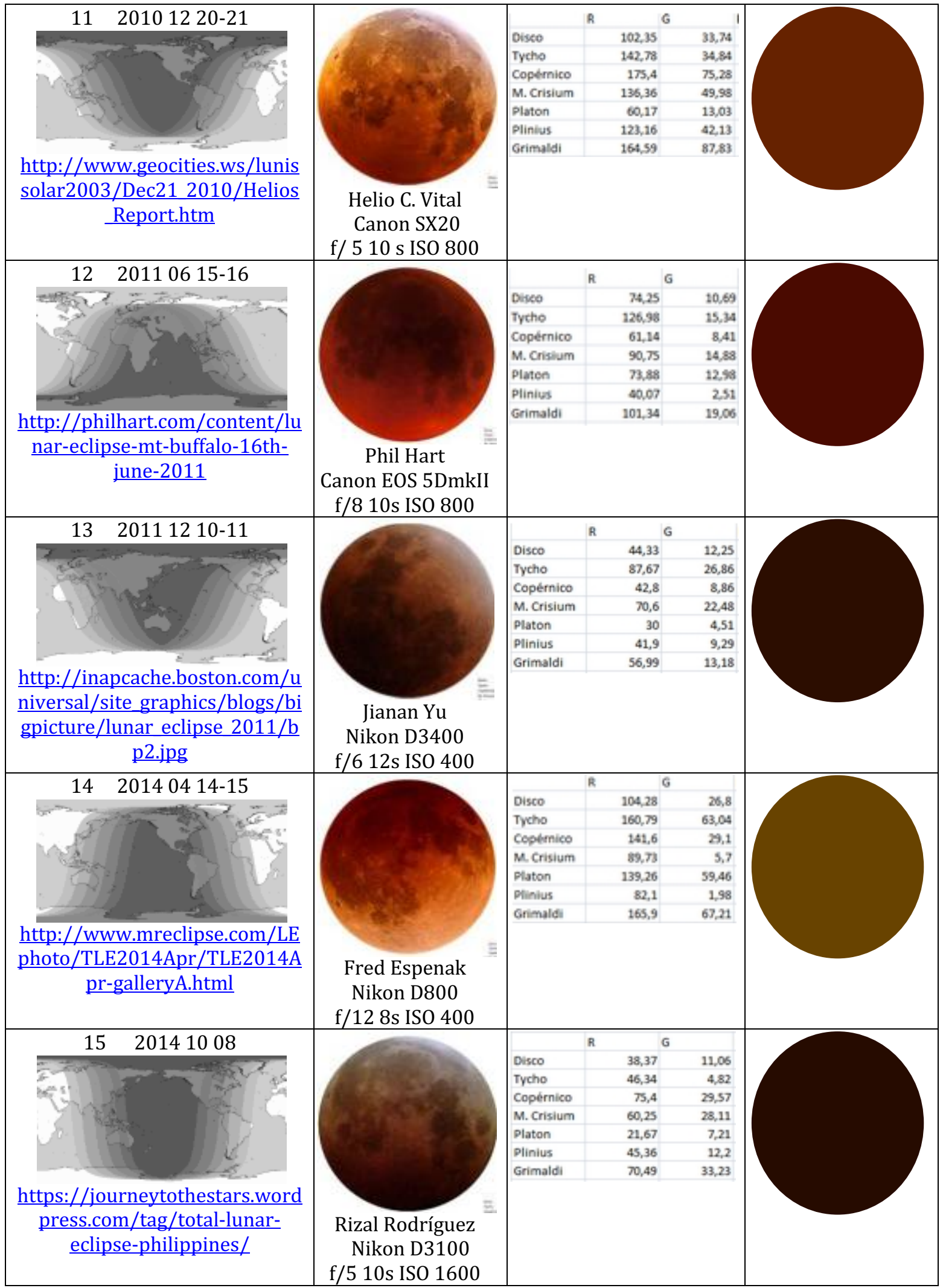




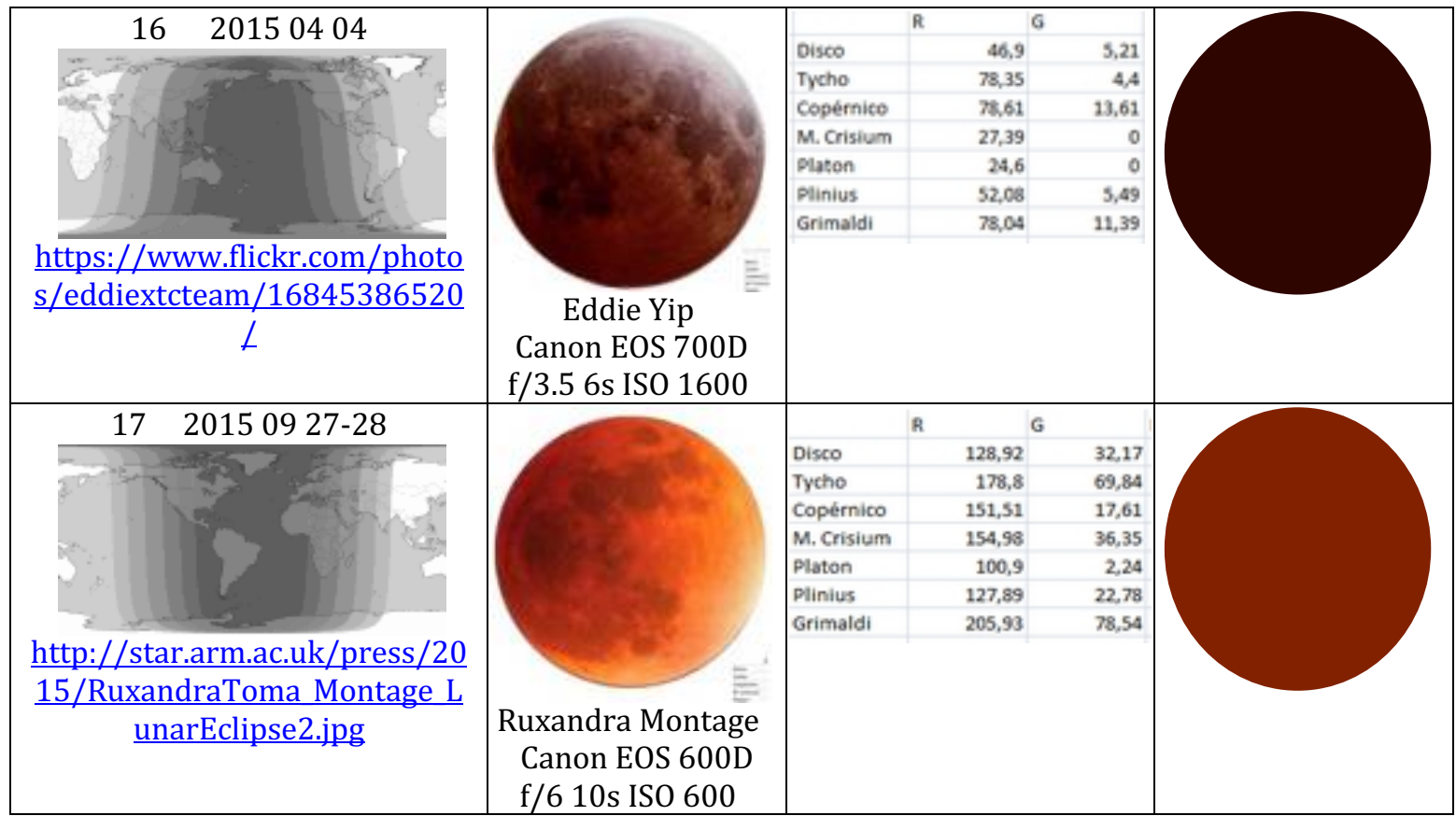

En la siguiente sección se usarán los valores de coordenadas cromáticas RG para evaluar el número de Danjon y la cromaticidad de los eclipses lunares estudiados.

\section{Resultados y Discusión}

La distribución de las medidas de cromaticidad de los accidentes lunares ocupan un subespacio acotado en el plano RG como se observa en la figura 6; con valores tales que $\mathrm{G}<100$ y $\mathrm{R}<2 \mathrm{G}$.

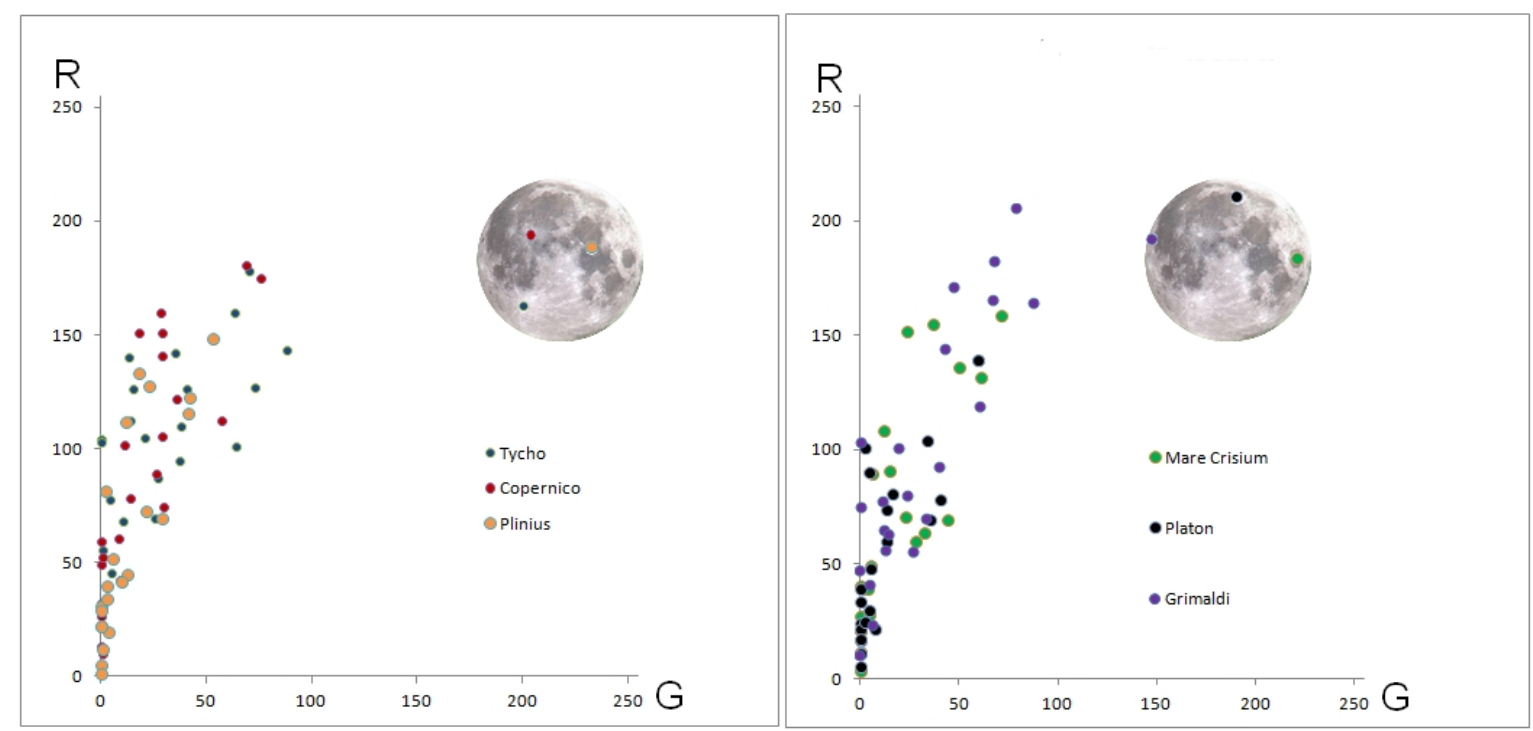

Fig. 6. Distribución en el plano RG de las imágenes de aéreas seleccionadas del disco lunar de los 17 eclipses lunares de la Tabla I: Cráteres brillantes (izq) zonas opacas (derecha).

En la figura 7 se muestran las componentes R y G para cada elipse particular (eventos 1 al 17 según la Tabla 1) para los accidentes lunares seleccionados, y se compara con los valores L0,L1, L2, L3 y L4 de la escala patrón de Danjon [12]. Se observa que un mismo eclipse (igual numeral en el eje de las abscisas) presentara diferentes número de Danjon si se compara individualmente con la escala patrón; principalmente por las propiedades de reflectividad de cada accidente lunar considerado y por la iluminación heterogénea del disco lunar eclipsado como se mostró en la Tabla I. 
La comparación del promedio de los valores RG de las (06) áreas correspondientes a los accidentes lunares seleccionados y su comparación con la escala patrón se muestra en la figura 8 (superior) junto al valor medio del disco total eclipsado (inferior). Se observa que ambas distribuciones son similares para cada evento particular, aun cuando los valores RG son nominalmente superiores en un $10 \%$ en los promedios respecto a los valores $\mathrm{R}$ del disco, quizá debido al oscurecimiento del limbo lunar. Igualmente se destaca que no es posible establecer una correspondencia univoca entre los valores RG de cada evento con los niveles RG de la escala de Danjon patrón.
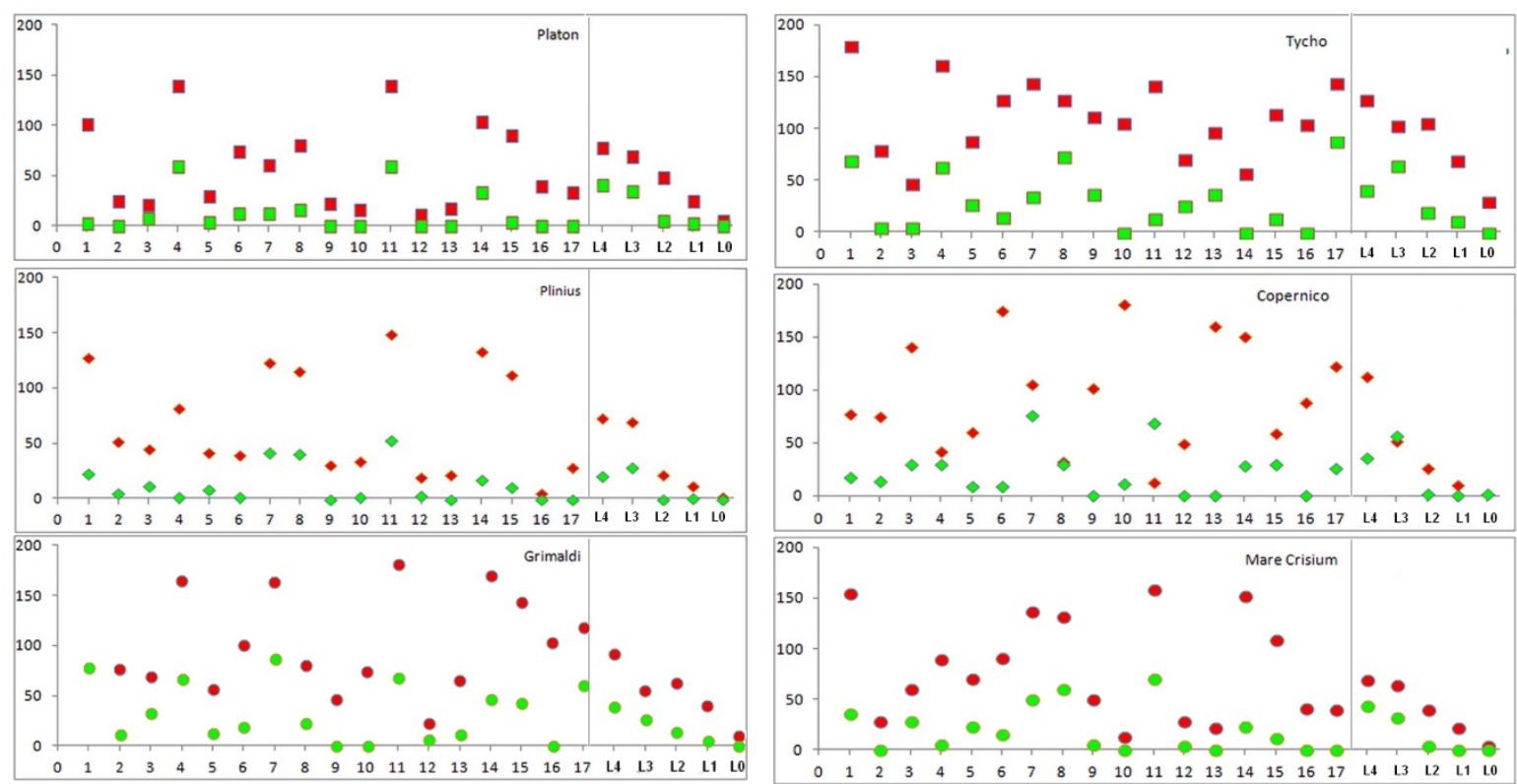

Fig. 7. Distribución de valores RG por Eclipses (eventos 1 al 17 según la Tabla1) y por accidentes lunares respecto a los valores RG de la escala Danjon patrón (L0-L4).

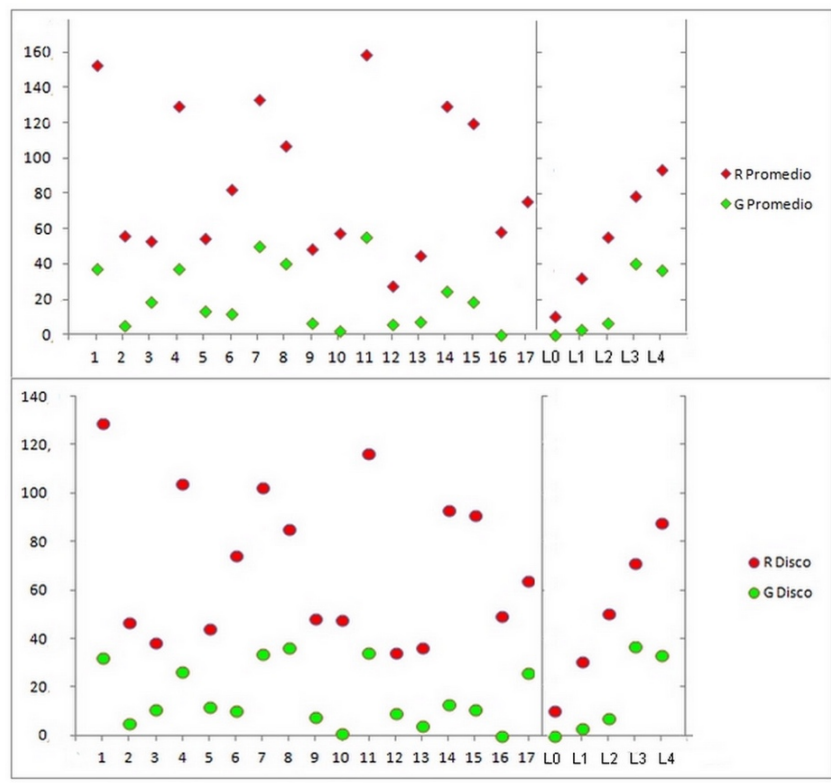

Figura 8 Distribución de coordenadas RG por evento y su comparación con la escala Danjon. Promedio de aéreas seleccionadas (superior) y Disco lunar completo (inferior).

Para evaluar la magnitud del eclipse y comparar con la escala patrón de Danjon, debemos observar la distribución de los eventos en el plano RG, como se muestra en la figura 9 (Izq). Se muestra que la distribución de los eventos en el plano $R G$ ocupan un subespacio limitado por las rectas $R=2 G$ y $R=2 G+90$, tanto para el disco completo (rombos azules) como para el promedio de las áreas seleccionadas (rombos rojos); los discos sombreados muestran los colores de referencia cromáticas. La escala patrón de Danjon en 
este gráfico no es lineal, sin embargo existe una correspondencia entre la magnitud del vector del espacio cromático de cada punto y el valor correspondiente a L0, L1, L2, L3 y L4. Los puntos más cercanos al origen resultan más oscuros y los más distantes resultan más brillantes; téngase en cuenta las coordenadas cromática de la figura 5: negro $(0,0,0)$ y amarillo digital $(255,255,0)$. La distribución por eventos se muestra en la parte derecha de la Fig. 9; donde se han dibujado también líneas isocromáticas como arcos de longitudes 0,098. Luego puede hacerse una correspondencia entre los eclipses (eventos) y la escala Danjon patrón por medio del módulo del vector cromático.
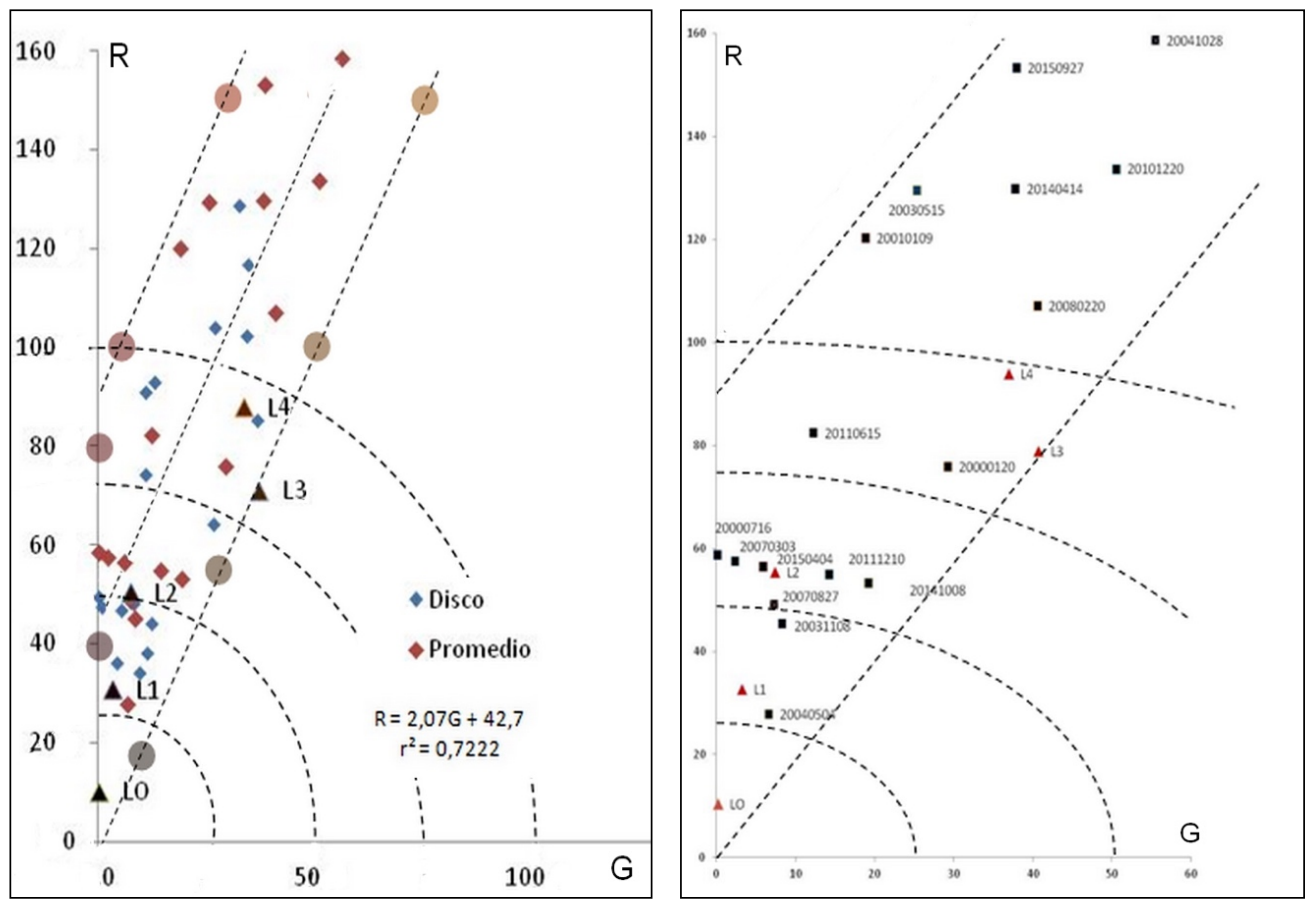

Figura 9 Distribución de cromaticidad RG de los Eclipses Totales de Lunar. Tendencia global (Izq.) por eventos (der).

Para ello se construye un número, denominado Factor Danjon, definido como el módulo normalizado del vector cromático en el plano RG:

$$
F D \equiv \frac{\sqrt{R^{2}+G^{2}}}{255 \sqrt{2}}
$$

La estimación del Factor de Danjon para los eventos 1-17 y para la escala Danjon patrón se presenta en la figura 10. El número FD permite estimar unívocamente la magnitud del eclipse total de Luna; e incluso los rangos del número FD permiten una clasificación en términos de la escala de Danjon original. Así resulta que no ocurrieron eclipse L0 en el periodo 2000-2017; solo tres eventos L1, cinco L3, dos L2 y cinco con L4 propiamente dicho; además se hace notar que los eclipses del 2000-01-20 y del 2010-12-20 fueron incluso más brillantes que los eclipses L4 usuales. 


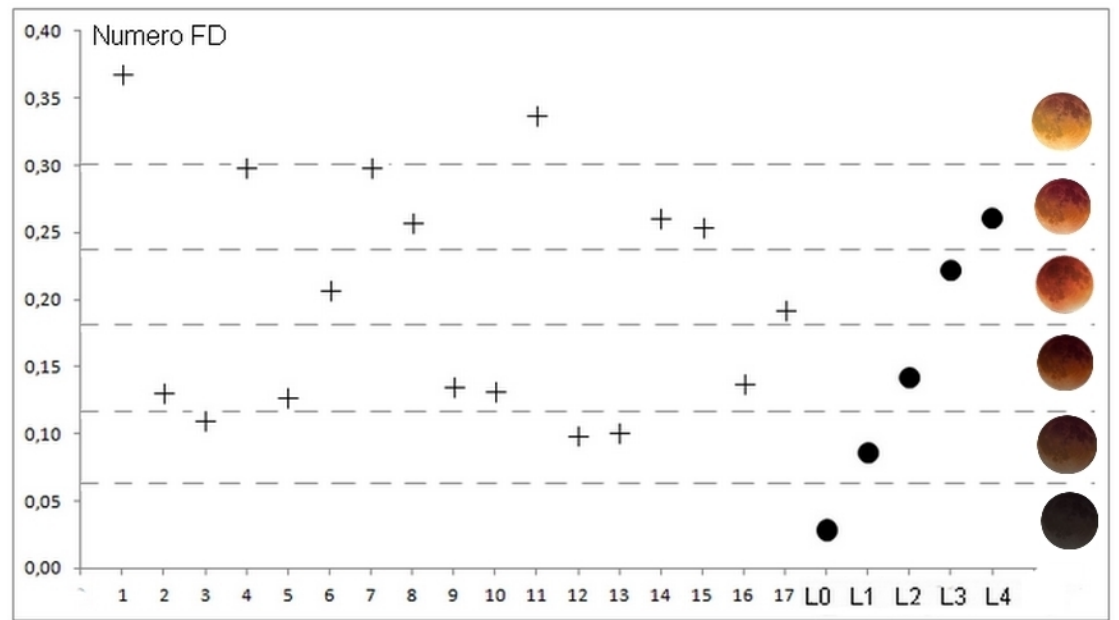

Figura 10 Distribución del Factor Danjon (FD) para los eventos estudiados y para la escala Danjon patrón. A la derecha imágenes alegóricas de la apariencia del disco lunar eclipsado. Fuente: los Autores.

\section{Conclusiones}

Como se discutió en las figuras 3 y 4 las longitudes del cono de sombra proyectado sobre la luna eclipsada, durante la fase del máximo, varía desde valores inferiores a la distancia Tierra- Luna hasta valores tres veces superior a ésta; es diferente para cada longitud de onda en el intervalo 500-700 nanómetros de la región visible del espectro electromagnético, y la transmitancia depende del tamaño y concentración de los aerosoles terrestre. Esta variación de la longitud del cono de sombra cromático, proyectado sobre la Luna Eclipsada, puede relacionarse fenoménicamente con la escala de Danjon para describir la magnitud y el color del disco lunar durante el máximo del eclipse total de Luna.

Los registros fotográficos en el periodo 2000-2017, de la fase de máximo en los eclipses totales de Luna presentan una variación no regular en la magnitud de brillo aparente y en la cromaticidad, en periodo incluso inferiores a seis meses para los eventos ocurridos en un mismo año: eventos 4-5 año 2003, 6-7 año 2004, 8-9 año 2007, 12-13 año 2011, 14-15 año 2014 y 16-17 año 2017 (Tabla 1). Vale decir, los registros de la magnitud del brillo aparente y de la cromaticidad, es tal que no parece vinculada a las circunstancias astronómicas del eclipse total, ni a la posición del observador, ni a ciclos naturales orbitales del movimiento Tierra-Luna o Tierra-Sol.

Las figuras 6 y 9 evidenciaron que el color RGB de las imágenes digitales de la Luna eclipsada en el máximo, presentan valores de rojo (R) y verde (G) siguiendo la relación media $R=2 G$ y componente azul (B) nula o depreciable (en comparación a los niveles RG en las imágenes no balanceadas cromáticamente); en concordancia a la selectividad cromática prescrita por la relación de dispersión de Rayleigh (Ecuación 3).

Los valores RG promediados sobre el disco lunar completo o promediados a partir de una muestra de accidentes lunares significativos presentan la misma variación cromática para los eventos estudiados, como se mostró en las figuras 8 y 9; y en todo caso, ambos promedios no guardan correlación con la escala Danjon patrón [12]. Huelga decir que los accidentes topográficos lunares (Copérnico, Tycho, Platón, Grimaldi, Plinius y Mare Crisium) empleados como testigos son una muestra confiable y suficiente para estimar la escala de Danjon Patrón [12] y el factor de Danjon, ecuación (7).

La distribución de los eventos y la escala Danjon en el plano RG permite vincular el brillo aparente con el color a partir del módulo normalizado del vector cromático, por medio de la ecuación (7); que hemos denominado Factor de Danjon; resulta una medida eficaz y univoca, de la magnitud y cromaticidad en los Eclipses Totales de Luna.

\section{Agradecimientos}

Se agradece la solidaridad de la Sociedad Española de Óptica (SEDOPTICA) y al Comité Editorial de la Revista Óptica Pura y Aplicada por la publicación. Los Autores agradecen las observaciones y comentarios de un Árbitro anónimo que mejoró sustancialmente el manuscrito. 